Beliefs about Control and the Persistence of Cleaning Behaviour: An Experimental Analysis

\author{
Laurie A. Gelfand ${ }^{\mathrm{a}} \&$ Adam S. Radomsky ${ }^{* a}$ \\ Concordia University
}

${ }^{\mathrm{a} C o n c o r d i a}$ University

Department of Psychology

7141 Sherbrooke St. W.

Montreal, QC, Canada, H4B 1R6

* Corresponding Author. Tel.: +1 514848 2424/2202; fax: +1 5148484523. E-mail address: adam.radomsky@concordia.ca (A.S. Radomsky) 


\begin{abstract}
Background and Objectives: Low perceived control (PC) and overestimations of controllability have each been related to obsessive compulsive (OC) symptoms and behaviour. OC beliefs and symptoms are also associated with a discrepancy between low perceived control (PC) and a high desire for control (DC). The present study sought to examine the influence of components of PC, low control-related self-efficacy (CSE) and high predicted controllability (PRC), on the persistence of cleaning behaviour and DC ratings. Methods: A cleaning task was used to observe cleaning time (in seconds) in undergraduate participants $(n=174)$ under two conditions of each of PRC (high versus low), and CSE (high versus low). DC ratings were taken prior to the cleaning task. Results: It was demonstrated that PRC and CSE manipulations had differential effects on cleaning times and DC ratings, where significantly longer cleaning times were observed in the high (versus low) PRC condition, and in association with higher DC ratings reported in the low (versus high) CSE condition. However, regression analyses demonstrated that DC, PRC and CSE each accounted for significant variance in observed cleaning times.

Limitations: Teasing apart predictability from controllability is a methodological challenge in the manipulation of perceived control. Conclusions: Findings highlight the importance of considering components of PC along with DC in OC-phenomenology; these will be discussed in the context of current cognitive theories of and treatments for OCD.
\end{abstract}

KEYWORDS: perceived control; illusion of control; self-efficacy; desire for control; OCD; contamination. 


\section{Beliefs about Control and the Persistence of Cleaning Behaviour: An Experimental}

\section{Analysis}

Research on perceived control (PC) and anxiety has demonstrated that low PC is an important factor in the aetiology and maintenance of anxiety disorders in general (Chorpita \& Barlow, 1998), as well as in association with specific conditions (Brown, White, Forsyth, \& Barlow, 2004; Cloitre, Heimberg, Liebowitz, \& Gitow, 1992; Hoffman, 2005; White, Brown, Somers, \& Barlow, 2006; Zvolensky, Lejuez, \& Eifert, 2000). For example, low PC has been implicated in the maintenance of symptom severity in social phobia (e.g., Hofmann, 2005), panic disorder (e.g., White et al., 2006), pathological gambling (Goodie, 2005), as well as obsessivecompulsive disorder (OCD; Moulding \& Kyrios, 2006). In OCD, low PC is thought to contribute to the urge to engage in repetitive behaviour specifically when it co-occurs with a high desire for control (DC; Moulding, Doron, Kyrios, \& Nedeljkovic, 2008; Moulding \& Kyrios, 2006, 2007), such that compulsive behaviour is motivated by a desire to (re)establish a sense of control over anxiety-related outcomes (Reuven-Magril, Dar, \& Liberman, 2008). While common characterizations of OCD have often included notions of control or controllability of emotion, behaviour, events and/or objects, theoretical models and empirical explorations of control in OCD have generally been limited to the control of thoughts (Purdon \& Clark, 2002; Obsessive Compulsive Cognitions Working Group [OCCWG], 2005; Tolin, Woods, \& Abramowitz, 2003). The present study therefore sought to examine and clarify the function of perceived control beliefs and desire for control in association with OC-phenomenology.

Current cognitive models of OCD (e.g., Rachman, 1997, 1998, 2002; Salkovskis, 1985, 1999) suggest that individuals with OCD become anxious due to the misappraisal of normal intrusive thoughts as overly significant, and engage in compulsive behaviour in direct response 
to those misappraisals, thus relieving anxiety by decreasing the perceived likelihood of future negative outcomes. Compulsive behaviour is thought to strengthen the frequency and intensity of intrusive thoughts by supporting, if not reinforcing the misappraisals, which in turn will lead to increased compulsive behaviour. Three belief domains have been found to be related to the misappraisal of intrusive thoughts in OCD: 1) inflated responsibility and threat estimation, 2) perfectionism and intolerance of uncertainty, and 3) importance and control of thoughts, and are assessed via the Obsessive Beliefs Questionnaire (OBQ - OCCWG, 2005). A significant proportion of individuals with OCD however appear not to report high levels of the aforementioned belief domains on the OBQ (Calamari et al., 2006; Taylor et al., 2006), which suggests that for some individuals, other belief domains may be particularly relevant to the misappraisal of intrusive thoughts in OCD. Investigations of control-related beliefs (Moulding \& Kyrios, 2007; Moulding et al., 2008; Moulding, Kyrios, Doron, \& Nedeljkovic, 2009) demonstrate that beliefs about control may a viable addition to explain a greater amount of shared variance than is accounted for by existing belief domains.

There are two general approaches to the scientific study of control. First, it is thought that the subjective experience of control (i.e., PC) is of greater importance to mental and physical health outcomes than any objective fact of controllability (Lazarus \& Folkman, 1984; Skinner, 1996). However, PC is a considered a difficult concept to investigate methodologically most likely because it presents a significant challenge in definition (Skinner, 1996). In OCD research, PC is thought to involve such constructs as self-efficacy (Moulding \& Kyrios, 2006) and overestimations of control (Reuven-Magril et al., 2008). Second, it is thought that people vary in the degree to which they desire to seek and maintain a sense of control and often behave ways that promote this likelihood, even when it is potentially maladaptive (Deci \& Ryan, 2000; 
Zuckerman, Knee, Kieffler, Rawthorne, \& Bruce, 1996). Research on PC, DC and OCD has revealed that a "control mismatch" (i.e., high DC/low PC) is related to OC-symptoms, beliefs, and behaviour, wherein the discrepancy between the control appraisals is thought to be an important factor in maintaining the urge to engage in repeated behaviour (Moulding \& Kyrios, 2007; Moulding et al., 2008, 2009). The present research is concerned with clarifying the role of PC in OC phenomenology in the context of the "control mismatch" of PC and DC.

The relationship between between control-related self-efficacy (CSE) appraisals and OCbeliefs and symptoms in both non-clinical and clinical samples has been examined by Moulding and colleagues $(2007,2008)$. Participants read hypothetical scenarios of an OC-relevant event (a dripping tap) in which both level of threat (high/low) and responsibility (high/low) were manipulated, and were asked to assess their level of PC and DC, as well as responsibility, threat, affect and (desire to take) action. In both studies, results indicated that DC was found to increase with threat and responsibility, and that together high DC and low PC were associated with OCphenomenology (e.g., negative affect, the propensity to act in relation to threat) over and above such cognitions as inflated responsibility. Levels of PC and sense of control appraisals were consistently low in the high OC (2007) and OCD (2008) groups. This result suggests that a (low) sense of CSE, when combined with an increasing desire for control, may result in increased OC symptoms (Moulding \& Kyrios, 2007). The findings suggest that in spite of the association of low PC with responsibility and threat beliefs found in earlier OCD research (Moulding \& Kyrios, 2007), responsibility, threat, and control cognitions appear to be distinct from each other and may not vary together in a phenomenological manner. A low sense of control may thus be characteristic of individuals with OCD, and possibly comprised of CSE cognitions differentiable from other OC-beliefs. 
Reuven-Magril and colleagues (2008) investigated the relationship between OC symptoms and the overestimation of control. Using an illusion-of-control paradigm, participants were presented with preprogrammed visual stimuli, and were told that they could shorten the presentation time of each item by pressing the right combination of keys (i.e., that the stimuli was controllable). False positive feedback on participants' control attempts (to enhance the overestimation of control) was given through gradual decreases of stimulus presentation time; ratings of control estimations were taken at three time points during the task. Results revealed that individuals with high OC tendencies were associated with higher estimations of control, increased behavioural attempts to control, as well as a more restricted range of control behaviours. This finding suggests that predictions of controllability (PRC) are involved in increasing the urge to engage in compulsive behaviour, and allows for the speculation that overstimations of control may be involved in heightening PC following repetitive behaviour. Manipulating PRC beliefs permits further testing of the influence of overestimations of control on OC-type behaviour, and targets the notion of predictability that is inherent in the construct of the overestimation of control.

In light of the burgeoning interest in the relationship between control beliefs and OCD, and despite a growing number of psychometric findings, there is a general paucity of experimental research examining the influence or effects of perceived control-related beliefs on OC-type behaviour. This first aim of the study was to examine the influence of manipulations of OC-specific control-related cognitions, PRC and CSE, on cleaning time and desire for control (DC) ratings. It was predicted that higher cleaning times and higher DC ratings would be observed in the high PRC/low CSE condition. The second aim of the study was to examine the influence of control-related cognitions and DC on cleaning time. It was hypothesized that 
manipulations of PRC and CSE would each be positively associated with cleaning time, and that these relationships would be moderated by DC. That is, DC ratings were speculated to influence the strength of the relationship between PRC/CSE manipulations and cleaning times.

\section{Method}

\section{Participants}

One hundred seventy-four volunteer undergraduate students from the Department of Psychology at Concordia University in Montréal, Canada, participated in this study. Participants' mean age was $24.01(S D=14.32)$ years, and $76.6 \%$ of participants were female. Participants were compensated for their time with either course credit or entry in a draw for a cash prize. Participants' scores on relevant self-report symptom measures (see below) are displayed in Table 1.

\section{Measures}

Beck Anxiety Inventory (BAI; Beck \& Steer, 1990) and Beck Depression Inventory-II (Beck, Steer, \& Brown, 1996). The BAI and BDI-II are widely used and well-validated 21-item self-report instruments for the assessment of state anxiety and depression, respectively. The BAI exhibits good internal consistency (Creamer, Foran, \& Bell, 1995; Fydich, Dowdall, \& Chambless, 1992), modest test-retest reliability (Creamer et al., 1995; Fydich et al., 1992), and excellent divergent validity in comparison with other measures of anxiety (Creamer et al., 1995; Fydich et al., 1992). The internal consistency within the current sample was excellent $(\alpha=.90)$. The BDI-II demonstrates high internal consistency and good test-retest reliability, as well as good convergent and divergent validity (Beck et al., 1996; Steer \& Clark, 1997). The internal consistency within the current sample was excellent $(\alpha=.92)$. 
Obsessive Beliefs Questionnaire-44 (OBQ-44; OCCWG, 2005). This 44-item scale is a revision of the OBQ-87, and is designed to assess beliefs and appraisals related to obsessional thinking. The OBQ-44 shows excellent internal consistency, and in an obsessive compulsive (OC) sample, the OBQ-44 correlated strongly with measures of checking and fears of contamination. The internal consistency within the current sample was excellent $(\alpha=.94)$.

Vancouver Obsessional Compulsive Inventory (VOCI; Thordarson et al., 2004). The VOCI is a 55-item self-report instrument designed to measure obsessive compulsive symptomatology. There are six component subscales assessing various symptoms and features that have been found to be associated with OCD: checking; contamination; hoarding; indecisiveness; just right; and obsessions. The VOCI possesses good inter-item reliability in student, community, OCD, and clinical control populations, as well as high test-retest reliability in clinical (Thordarson et al., 2004) and student (Radomsky et al., 2006) populations. The scale has also been shown to have excellent convergent and divergent validity (Radomsky et al., 2006; Thordarson et al., 2004). The internal consistency within the current sample was excellent ( $\alpha=$ $.95)$.

Subjective rating scale (e.g., Wolpe, 1958). Participants were asked to provide subjective ratings at a variety of time points during the experiment using a 0-100 scale. These types of rating scales are often used in clinical research and treatment (e.g., Wolpe, 1990) Participants were asked to rate their level of desire for control (DC) with the question "How much do you desire to reduce/remove the germs from the keyboard?” As an index of CSE, participants were asked "How much do you feel you were able to control the level of germs on the keyboard?" 
Cleaning time. Participants were videotaoped during the two timed cleaning tasks. Two researchers who were blind to participant condition viewed the recordings independently and transcribed participants' cleaning time (in seconds) onto coding sheets . The coders were trained to record cleaning time and behaviour by coding 6 randomly selected videotapes, using guidelines created by the first author. Coders were required to obtain a minimum of $95 \%$ interrater agreement with each other on all 6 recordings before they could begin coding for the study.

\section{Study Design}

This study employed a 2 (PRC condition) x 2 (CSE condition) between-participants design, in which predicted controllability of the cleanliness of a computer keyboard and controlrelated self-efficacy regarding contamination control were experimentally manipulated. There were four randomly assigned conditions and in each, participants were exposed to one PRC manipulation and one CSE manipulation. DC ratings were collected following the CSE manipulation. The dependent variable was time spent cleaning the keyboard, which was video recorded for subsequent coding.

\section{Procedure}

Participants were informed that they were taking part in a study about hygiene, and were first asked to complete an online questionnaire package comprised of the BAI (Beck \& Steer, 1990), the BDI-II (Beck et al., 1996), the OBQ-44 (OCCWG, 2005), and the VOCI (Thordarson et al., 2004). To manipulate PRC, partcipants were asked to read condition-specific (i.e., modified) versions of a newspaper article containing information on hygiene in the workplace ("Lifting the Lid on Computer Filth", 2004). The high PRC group read that “...individuals can expect to be able to control germs up to $97.5 \%$..." and the low PRC group read "...up to 33.3\%..." (emphasis added). Participants were then give a three question multiple choice quiz 
within which was a question that asked by what percentage the spread of contamination can be reduced. The four reponse options were: "it cannot be reduced", $33.3 \%, 97.5 \%$, and 100\%. Participants were then trained to "properly" clean a computer keyboard and subsequently asked to clean the keyboard according to the training protocol (Cleaning Time 1). As the CSE manipulation, participants were presented with one of two false data outputs regarding the status of keyboard contamination. In the high CSE condition, participants received feedback that they had successfully removed most of the contamination, while in the low CSE condition, participants received feedback that they not successfully removed most of the contamination. Participants were asked to provide subjective CSE ratings following the feedback as a manipulation check.

Participants were told that there would be a few additional questionnaires to complete on the computer, and the experimenter removed the cleaned and assessed keyboard and replaced it with a second one that was visibly dirty. The experimenter left the room and indicated that if the participant so desired s/he could clean the keyboard before finishing the last few online measures (Cleaning Time 2). When the experimenter returned after one minute, and if the participant indicated that they wished to continue cleaning, they were left to do so. Once the participants indicated that they had finished cleaning, participants were debriefed as to the objectives of the study and informed of the deception involved in the study. They were asked to provide consent to the use of their data given the deception used in the study.

\section{Results}

\section{Participant Characteristics}

Participants in the four conditions did not differ with respect to age, $F(3,170)=1.13, p=$ .34 , partial $\eta^{2=} .003$ nor did they differ in terms of their mean total scores on the VOCI, OBQ-44, 
the BAI or the BDI-II, all $F_{s}(3,173) \leq 2.25, p \mathrm{~s} \geq .1$, partial $\eta^{2} \mathrm{~s} \leq .03$. A chi-squared analysis revealed that proportion of males to females did not differ significantly across the four conditions $\left(\chi^{2}[1, \mathrm{~N}=174]=2.77, p=.6\right.$.

\section{Missing Data}

The data from 12 participants were removed due to technical difficulties regarding their video recordings. Analyses were conducted on the data from the remaining 174 participants.

\section{Manipulation checks}

To first determine if the experimental manipulations of PRC and CSE were successful, $t$ tests were employed using the manipulation check ratings. The PRC manipulation check revealed no significant differences between the two PRC groups in terms of correct responses to the manipulation check question, $t(173)=-1.02, p=.31, d=.1$, as all but 1 participant (assigned to the low PRC condition) provided the correct answer relevant to their condition assignment. As predicted, participants in the low CSE condition rated their ability to control the germs on the keyboard as significantly poorer than participants in the high CSE condition, $t(173)=11.22, p<$ $.001, d=1.7(M=39.86[S D=28.77]$ vs. $M=79.03[S D=15.76])$.

\section{Coder reliability}

In order to assess the coder reliability of cleaning time, cleaning time (in seconds) was compared between coders for $24 \%$ of the sample. Inter-rater agreement was excellent for cleaning time $(99 \%)$ in the comparison sample.

\section{Main analyses}

For the hypothesis regarding cleaning time, two univariate ANOVAs were conducted on the data. For both ANOVAs, PRC and CSE were the between-participants factors. For the first 
ANOVA, cleaning time (in seconds) post-cleaning training (Time 1) was the dependent variable. For the second ANOVA, the optional final cleaning time (Time 2) was the dependent variable. For the first ANOVA, results revealed no significant main effects nor an interaction between the conditions on cleaning time during Cleaning Time $1, F \mathrm{~s}(3,171) \leq .41, p \mathrm{~s} \geq .33$, partial $\eta^{2} \mathrm{~s} \leq .004$. The second ANOVA (Cleaning Time 2) revealed that there was a significant main effect of PRC, $F(3,171)=4.06, p=.05$, partial $\eta^{2}=.02$, with the high PRC condition demonstrating longer cleaning times $(M=144.58, S D=196.62)$ than the low condition $(M=$ 95.01, $S D=120.21$ ) when collapsed across CSE conditions (see Figure 1). Contrary to expectations, there was no significant main effect for CSE nor a significant interaction between the conditions, $F \mathrm{~s}(3,171) \leq 4.06, p \mathrm{~s} \geq .29$, partial $\eta^{2} \mathrm{~s} \leq .006$ on cleaning time during Cleaning Time 2.

For the hypothesis regarding DC ratings, a univariate ANOVA was conducted. PRC and CSE were the between-participants factors, and pre-Cleaning Time 2 DC rating was the dependent variable. The ANOVA revealed that there was a significant main effect of CSE, $F(3$, $171)=33.65, p<.001$, partial $\eta^{2}=.16$, with the low CSE condition demonstrating higher DC ratings $(M=56.94, S D=34.99)$ than the high condition $(M=26.42, S D=34.24)$ when collapsed across PRC conditions (see Figure 2). Contrary to expectations, there was no significant main effect for PRC nor a significant interaction between the conditions, $F_{\mathrm{S}}(3,171) \leq .67, p \geq .42$, partial $\eta^{2} \mathrm{~s} \leq .004$ on DC ratings.

To test the hypothesis that PRC, CSE, DC ratings and their interactions would each uniquely predict time spent cleaning the keyboard at Cleaning Time 2, a hierarchical regression analysis was conducted. DC ratings and cleaning time data was centered prior to the analysis. PRC and CSE condition assignment were dummy coded and entered in the first step, and their 
product entered in the second step. In the third step, DC was entered, followed by the products of PRC and DC and CSE and DC in the fourth step. In the fifth and final step, the product of PRC, CSE and DC was entered. Time 2 cleaning time (in seconds) was the criterion variable. When entered in the first step, PRC and CSE combined did not account for any significant variability. When examined separately however PRC and CSE conditions were differentially related to time spent cleaning such that PRC was a significant predictor, $\beta=49.58, p=.05$, whereas CSE was not a significant predictor, $\beta=.123, p=.99$ When entered in the second step, the interaction term did not account for any additional variability. In the third step, DC accounted for an additional $7.9 \%$ of variability in time spent cleaning, $F \Delta(1,170)=15.02, p<.001$. The two interaction terms (PRC by DC, CSE by DC) entered in the fourth step accounted for an additional $10.4 \%$ of variability, $F \Delta(2,168)=11.16, p<.001$; both interaction terms were significant predictors. Finally, the three-way interaction entered in the final step did not account for any additional variance, $F \Delta(1,167)=.86, p=.36$. Regression coefficients are presented in Table 2.

In order to understand the nature of the two interactions in the fourth step of the hierarchical regression (PRC and DC, CSE and DC), correlations were conducted between DC ratings and Time 2 cleaning times in the high and low PRC and CSE groups. In the low PRC group, ratings of DC were not related to time spent cleaning, $r=.05, p=.67$, whereas in the high PRC group, DC ratings were significantly related to cleaning times, $r=.40, p<.001$. In the low CSE group, ratings of DC were significantly related to time spent cleaning, $r=.44, p<.001$, whereas no such relationship was found in the high CSE group, $r=.04, p=.72$. Intercorrelations, means and standard deviations of time spent cleaning (in seconds) in the high and low conditions of PRC and CSE as a function of DC rating are presented in Table 3. 


\section{Discussion}

This study aimed to investigate the influence of control-related cogntions on the persistence of cleaning behaviour. It was predicted that manipulating predicted controllability and control-related self-efficacy beliefs would influence time spent cleaning and desire for control ratings, such that high predicted controllability and low control-related self-efficacy would result in longer cleaning times and higher desire for control ratings. It was also hypothesized that the relationship of both control-related cogntions with desire for control ratings would predict longer cleaning times. As expected, the findings revealed that predicted controllability was directly related to time spent cleaning in that high predictions of controllability over a contamination threat led to longer cleaning times than low predicted controllability. While the CSE manipulation did not influence cleaning times, control-related self-efficacy manipulations were directly related to differences in desire for control ratings. That is, while DC ratings just prior to the final cleaning task were significantly higher in the low CSE group in comparison to the high group, there were no significant differences in DC ratings in either the PRC condition alone, nor was there a significant interaction between PRC and CSE. Finally, as hypothesized, and consistent with research implicating both DC and PC in OCphenomenology (Moulding \& Kyrios, 2007; Moulding et al., 2008, 2009), desire for control appraisals uniquely and in combination with PRC and CSE manipulations, significantly predicted time spent cleaning the keyboard. Correlational analyses revealed that DC was positively correlated with high PRC and with low CSE in the prediction of time spent cleaning. Overall, the results of the present study therefore demonstrate the importance of control-related cognitions in extended cleaning behaviour. 
Specifically, the present research suggests that inflated beliefs about controllability may contribute to extended cleaning behaviour, and provides preliminary evidence that these beliefs may underlie the urge to engage in compulsive behaviour (as was demonstrated by ReuvenMagril et al., 2008) and possibly reinforce it. That is, such beliefs may promote repeated behaviour in that individuals who overestimate controllability may then seek, in the context of a personally significant contamination threat, to reduce the discrepancy between high expectations of control and outcome controllability with repetitive behaviour. Repeated cleaning behaviour may therefore be in part an attempt to match inflated predictions of controllability with the expected outcome in order to relieve anxiety.

That low control-related self-esteem had an influence on desire for control ratings but not on cleaning times serves to broaden the basis of support for two important considerations in studying control in general, as well as in relation to OCD. First, this result highlights the specific influence of control-related self-efficacy appraisals on levels of DC, and thus provides further evidence on the necessity of considering PC and DC together in investigations of control-related cognitions. Second, that CSE had an effect on DC ratings but not on cleaning times suggests that control-related self-efficacy is a necessary, but not sufficient component of PC as it exists within the "control mismatch" in OCD. This finding thus supports the conceptualization of low PC in OCD as a composite variable (e.g., Moulding \& Kryios, 2006; Skinner, 1996), and underscores the importance of unpacking the layers of control beliefs to determine the specific nature of faulty underlying control beliefs in OCD.

Results of the regression analysis extend the findings of previous research on controlrelated beliefs and OCD in that they provide evidence that both components of PC are related to cleaning time through the influence of DC. These results support the notion that control-related 
beliefs in OC- behaviour are multifaceted in composition in that both overestimations of controllability and low control-related self-efficacy predicted longer extended cleaning behaviour in the presence of high DC. These findings also demonstrate evidence of a control mismatch in the prediction of cleaning time, in that low levels of control-related self-efficacy were related to longer cleaning when moderated by DC. While underlying inflated beliefs of controllability may lead to extended cleaning behaviour, the presence of high DC may increase the persistence of such behaviour. In fact, motives such as a high desire for control have been found to affect judgements of control such that desire for an outcome may result in overestimating the chances of influencing an outcome (Thompson et al., 2004). DC was the only variable that, on its own, accounted for a significant proportion of the variance in the prediction of time spent cleaning. DC may well be the critical ingredient in the functioning of control mismatch in OCD. That both components of PC were stronger predictors of time spent cleaning when considered concurrently with DC speaks to the importance of assessing DC in OCD.

As proposed in cogntive theories of OCD (e.g., Rachman, 1998; Salkovskis, 1999), the misappraisal of intrusive thoughts as personally significant and anxiety provoking due to underlying faulty beliefs is thought to lead to compulsive behaviour. Although three belief domains have been identified by researchers as specifically relevant to OCD (OCCWG, 2005), these beliefs do not fully account for all negative interpretations of intrusions nor maladptive behavioural responses (i.e., compulsions). Newer cognitive approaches have proposed that incorporating beliefs about the self and the world would enhance our understanding of the phenomenology of OCD, and more specifically that discrepant world- and self-controllability beliefs are involved in the development of OCD (Doron, Kyrios, \& Moulding, 2007a; Doron, Kyrios, Moulding, Nedeljkovic, \& Bhar, 2007b). The results of the present study provide 
preliminary evidence of the importance of including self-and world-concepts into cognitive theories of OCD in that both high predictions of controllability (i.e., world-controllability) and low control-related self-esteem (i.e., self-controllability) beliefs appeared to be involved in changing levels of PC over time.

\section{Limitations}

Importantly, the findings need to be interpreted in the context of a number of limitations. First, while OC-symptomatology and beliefs have commonly been found to be present in the general population (Gibbs, 1996; Muris, Harald, \& Clavan, 1997; Rachman \& de Silva, 1978), and although analogue research is common in examinations of OCD, it is possible that the current findings are an example of the differences between a clinical and non-clinical sample rather than indicative of the dimensional nature of OC-phenomenology. However, cleaning behaviour was chosen as the main outcome variable because it is a normative behaviour and thus amenable to analogue research. Second, both over-prediction of fear (Rachman, 1994) and overestimations of controllability (Reuven-Magril et al., 2008) appear to be characteristic of individuals with OCD. In the present study, it is not possible to determine whether it was the predictability or the controllability of the hygiene information that was responsible for the observed changes in behaviour in the present study. This suggests that teasing apart the individual and interaction effects of predictability versus controllability is a methodological necessity in the overall investigation of perceived control beliefs that needs to be addressed in future research. Lastly, during data collection for this study, there was widespread global concern regarding an H1N1 (Influenza A) virus pandemic. It is possible that ceiling effects due to this crisis were responsible for the present findings, as observing cleaning behaviour in the presence of a genuine contamination threat may limit the generalizability of these results. 


\section{Directions for future research}

Although control cognitions have been found to be most strongly related to fears of contamination/washing compulsions (Moulding et al., 2009), future research should examine the relation of control beliefs to other repetitive behaviours as well as extend the findings by testing a clinical population. Additionally, testing an experimental pardigm wherein desire for control was manipulated along with components of PC would allow for improved understanding of the function of DC within the control mismatch in OCD. While the present study demonstrated that control appraisals may indeed be an important variable in OC-phenomenology, it would be also be beneficial to determine in an experimental manner how such beliefs may interact with other OC-relevant beliefs. It may be that an increased desire for control may heighten responsibility and threat appraisals in similar ways. Finally, it would be of interest to further examine the constructs involved in the conceptualization of PC from a psychometric perspective to determine whether they should be considered and measured as domain-specific or as more stable individual difference traits.

\section{Clinical Implications}

As cognitive therapy for OCD includes assessing and challenging faulty beliefs that underlie the occurrence of obsession and compulsions, addressing control-related beliefs along with other OC-specific beliefs in the presence of OC-symptoms and behaviours would allow for improved case conceptualization and individualized treatment. The notion of control has been noted to be a common manner in which OCD symptoms are explained by individuals in therapy (Moulding \& Kryios, 2006), and incorporating questions of control and controllability into assessment would likely encourage collaboration and trust. More specifically, these results suggest that it may help individuals with OCD to determine what aspects of control and 
controllability contribute to their low sense of control. Finally, determining with a client how components of PC interact with a discrepant high DC may help them to understand how urges to engage in repetitive behaviour may be yoked to self- and world-controllability beliefs. In general, helping clients to determine exactly what components of PC contribute to feelings of low control, and to what degree the need for control is excacerbating beliefs about control, could become a therapeutic target in the reduction of compulsive behaviours.

\section{Summary}

Low perceived control has long been associated in the research literature with various forms of anxiety, and yet has been nearly absent in cognitive theories of OCD. The current study was an investigation of manipulations of two components of perceived control and their influence on OC-type cleaning behaviour; we believe that this was among one of the first studies to examine components of PC in an experimental manner. The results of the present study echo previous research on control-related beliefs and OCD (e.g., Moulding \& Kyrios, 2007) in that it will be important to consider not only the concurrent relationship of both PC and DC, but also the underlying components of PC shown to be relevant to OCD. Taken together, it appears that both components of PC under investigation are likely involved in $\mathrm{OC}$ behaviour, and it will thus be beneficial to consider PC in OC behaviour as a multidimensional variable. These findings also allow for a broader understanding of the 'control mismatch' in OCD, and that the role of DC should be considered in a comprehensive understanding of the function of control-related beliefs in OCD. 


\section{Author Note}

This research was supported in part by a graduate fellowship from the Fondations de recherche en santé du Quebec (FRSQ) and by an operating grant from the Canadian Institutes of Health Research (CIHR); neither funding source was involved in the design/conduct of the study, nor in the dissemination of our results. We would like to thank Stephanie Lavoie, Kevin Barber, G. Michael Everett, and Katie Gordon-Green for their help with participant recruitment and data collection. 


\section{References}

Beck, A. T., \& Steer, R. A. (1990). Beck Anxiety Inventory Manual .

Beck, A. T., Steer, R. A., \& Brown, G. K. (1996). Manual for the Beck Depression Inventory-II .

Brown, T. A., White, K. S., Forsyth, J. P., \& \& Barlow, D. (2004). The structure of perceived emotional control: Psychometric properties of a revised Anxiety Control Questionnaire. Behaviour Therapy (35), 75-99.

Calamari, J. E., Cohen, R. J., Rector, N. A., Szacun_Shimizu, K., Riemann, B.C., \& Norberg, M.M. (2006). Dysfunctional belief-based obsessive-compulsive disorder subgroups. Behaviour Research and Therapy, 44, 1347-1360.

Chorpita, B. F., \& Barlow, D. H. (1998). The development of anxiety: The role of control in the early environment. Psychological Bulletin, 124 (3-21).

Cloitre, M., Heimberg, R. G., Liebowitz, M. R., \& Gitow, A. (1992). Perceptions of control in panic disorder and social phobia. Cognitive Therapy and Research , 16, 569-577.

Creamer, M., Foran, J., \& Bell, R. (1995). The Beck Anxiety Inventory in a non-clinical sample. Behaviour Research and Therapy, 33, 477-485.

Deci, E.L. \& Ryan, R.M. (2000). The "what" and "why" of goal pursuits: Human needs and the self-determination of behavior. Psychological Inquiry, 11, 227-268.

Doron, G., Kyrios, M., \& Moulding, R. (2007a). Sensitive domains of self-concept in obsessivecompulsive disorder (OCD): Further evidence for a multidimensional model of OCD. Journal of Anxiety Disorders , 21, 433-444.

Doron, G., Kyrios, M., Moulding, R., Nedeljkovic, M., \& Bhar, S. (2007b). "We Do Not See Things as They Are, We See Them as We are":A Multidimensional Worldview Model of 
Obsessive Compulsive Disorder. Journal of Cognitive Psychotherapy: An International Quarterly, 21, 217-231.

Fydich, T., Dowdall, D., \& Chambless, D. L. (1992). Reliability and validity of the Beck Anxiety Inventory. Journal of Anxiety Disorders , 6, 55-61.

Gibbs, N. (1996). Nonclinical populations in research on obsessive-compulsive disorder: A critical review. Clinical Psychology Review , 16, 729-773.

Goodie, A. S. (2005). The role of perceived control and overconfidence in pathological gambling. Journal of Gambling Studies, 21, 481-502.

Hoffman, S. (2005). Perception of control over anxiety mediates the relation between catastrophic thinking and social anxiety in social phobia. Behaviour Research and Therapy, 43, 885-895.

Lazarus, R. S., \& Folkman, S. (1984). Stress, Appraisal, and Coping. New York: Springer.

Lifting the Lid on Computer Filth. (2004, March 4). BBC News. Retrieved from http://news.bbc.co.uk/go/pr/fr/-/1/hi/health/3505414.stm.

Moulding, R., \& Kyrios, M. (2006). Anxiety disorders and control related beliefs: The exemplar of obsessive-compulsive disorder. Clinical Psychology Review , 26, 573-583.

Moulding, R., \& Kyrios, M. (2007). Desire for control, sense of control and obsessivecompulsive symptoms. Cognitive Therapy and Research, 31, 759-772.

Moulding, R., Doron, G., Kyrios, M., \& Nedeljkovic, M. (2008). Desire for control, sense of control and obsessive compulsive checking: An extension to clinical studies. Journal of Anxiety Disorders . 
Moulding, R., Kyrios, M., Doron, G., \& Nedeljkovic, M. (2009). Mediated and direct effects of general control beliefs on obsessive compulsive symptoms. Canadian Journal of Behavioural Science, 41, 84-92.

Muris, P., Harald, M., \& Clavan, M. (1997). Abnormal and normal compulsions. Behaviour Research and Therapy, 35, 249-252.

Obsessive Compulsive Cognitions Working Group (OCCWG). (2005). Psychmetric validation of the obsessive belief questionnaire and interpretation of intrusions inventory-Part 2: Factor analyses and testing of a brief version. Behaviour Research and Therapy , 43, 1527-1542.

Purdon, C., \& Clark, D. A. (2002). The need to control thoughts. In R. O. Frost, \& G. S. (Eds.) (Eds.), Cognitive Approaches to Obsessions and Compulsions: Theory, assessment and treatment (pp. 29-43). Oxford: Elsevier.

Rachman, S. (1994). The overprediction of fear: A review. Behaviour Research and Therapy, $32,683-690$.

Rachman, S. (1997) A cognitive theory of obsessions. Behaviour Research and Therapy, 35, 793-802.

Rachman, S. (1998). A cognitive theory of obsessions: Elaborations. Behaviour Research and Therapy, 36, 385-401.

Rachman, S. (2002). A cognitive theory of compulsive checking. Behaviour Research and Therapy, 40, 624-639.

Rachman, S., \& de Silva, P. (1978). Abnormal and normal obsessions. Behaviour Research and Therapy, 16, 233-248.

Radomsky, A. S., Ouimet, A. J., Ashbaugh, A. R., Lavoie, S., Parrish, C. L., \& O'Connor, K. P. (2006). Psychometric properties of the French and English versions of the Vancouver 
Obsessional-Compulsive Inventory and the Symmetry, Ordering and Arranging Questionnaire. Cognitive Behaviour Therapy, 35, 164-173.

Reuven-Magril, O., Dar, R., \& Liberman, N. (2008). Illusion of control and behavioral control attempts in Obsessive-Compulsive Disorder. Journal of Abnormal Psychology, 117, 334341.

Salkovskis, P. (1985). Obsessional-compulsive problems: A cognitive-behavioural analysis. Behaviour Research and Therapy, 23, 571-583.

Salkovskis, P. (1999). Understanding and treating obsessive compulsive disorder. Behaviour Research and Therapy, 37, S29-S52.

Skinner, E. A. (1996). A guide to constructs of control. Journal of Personality and Social Psychology, 71, 549-570.

Steer, R. A., \& Clark, D. A. (1997). Psychometric characteristics of the Beck Depression Inventory-II with college students. Measurement and Evaluation in Counseling and Development, 30, 128-136.

Taylor, S., Abramowitz, J. S., McKay, D., Calamari, J. E., Sookman, D., Kyrios, M., et al. (2006). Do dysfunctional beliefs play a role in all types of obsessive-compulsive disorder? Journal of Anxiety Disorders, 20, 85-97.

Thompson, S.C., Kyle, D., Osgood, A., Quist, R.M., Phillips, D.J., \& McClure, M. (2004). Illusory control and motives for control: The role of connection and intentionality. Motivation and Emotion, 28, 315-330.

Thordarson, D., Radomsky, A. S., Rachman, S., Shafran, R., Sawchuk, C. N., \& Hakstian, H. R. (2004). The Vancouver Obsessional Compulsive Inventory (VOCI). Behaviour Research and Therapy, 42, 1289-1314. 
Tolin, D. F., Woods, C. M., \& Abramowitz, J. S. (2003). Relationship between obsessive beliefs and obsessive compulsive symptoms. Cognitive Therapy and Research, 27, 657-669.

White, K. S., Brown, T. A., Somers, T. J., \& Barlow, D. H. (2006). Avoidance behavior in panic disorder: The moderating influence of perceived control. Behaviour Research and Therapy, 44, 147-157.

Wolpe, J. (1958). Psychotherapy by reciprocal inhibition. Stanford, CA: Stanford University Press.

Wolpe, J. (1990). The Practice of Behavior Therapy (4 ${ }^{\text {th }}$ ed.). New York, NY: Pergamon Press.

Zuckerman, M., Knee, C. R., Kieffer, S. C., \& Gagne, M. (1996). Beliefs in realistic and unrealistic control: Assessment and implications. Journal of Personality, 64, 435-464.

Zvolensky, M. J., Lejuez, C. W., \& Eifert, G. H. (2000). Prediction and control: Operational definitions for the experimental analyses of anxiety. Behaviour Research and Therapy, $38,653-663$. 
Table 1.

Participants' scores on self-report measures.

$$
M(\mathrm{SD})
$$

Condition

Low PRC / Low PRC / High PRC / High PRC / Total

Low CSE High CSE Low CSE High CSE

$(n=42) \quad(n=43) \quad(n=43) \quad(n=46) \quad(n=174)$

$\begin{array}{llllll}\text { BAI } & 11.10(9.38) & 7.95(8.49) & 10.51(7.38) & 12.93(10.81) & 10.66(9.23) \\ \text { BDI } & 10.62(9.45) & 7.00(6.51) & 8.98(6.70) & 10.72(10.17) & 9.26(8.48) \\ \text { OBQ } & 137.95(36.97) & 125.81(40.6) & 127(32.96) & 134.8(38.17) & 131.41(37.32) \\ & & & & & \\ \text { VOCI } & 36.62(26.7) & 29.72(28.49) & 29.81(19.82) & 39.17(33.96) & 33.91(27.91) \\ & & & & & \end{array}$

Note: $\mathrm{BAI}=$ Beck Anxiety Inventory, BDI = Beck Depression Inventory, $\mathrm{OBQ}=$ Obsessive Beliefs Questionnaire, VOCI = Vancouver Obsessional Compulsive Inventory, VOCI clean $=$ cleaning subscale of the Vancouver Obsessional Compulsive Inventory, PRC $=$ Predicted controllability condition, CSE $=$ Control-related self-efficacy condition. 
Table 2.

Summary of Hierarchical Regression Analysis for PRC, CSE and DC Predicting Time 2

Cleaning (in seconds)

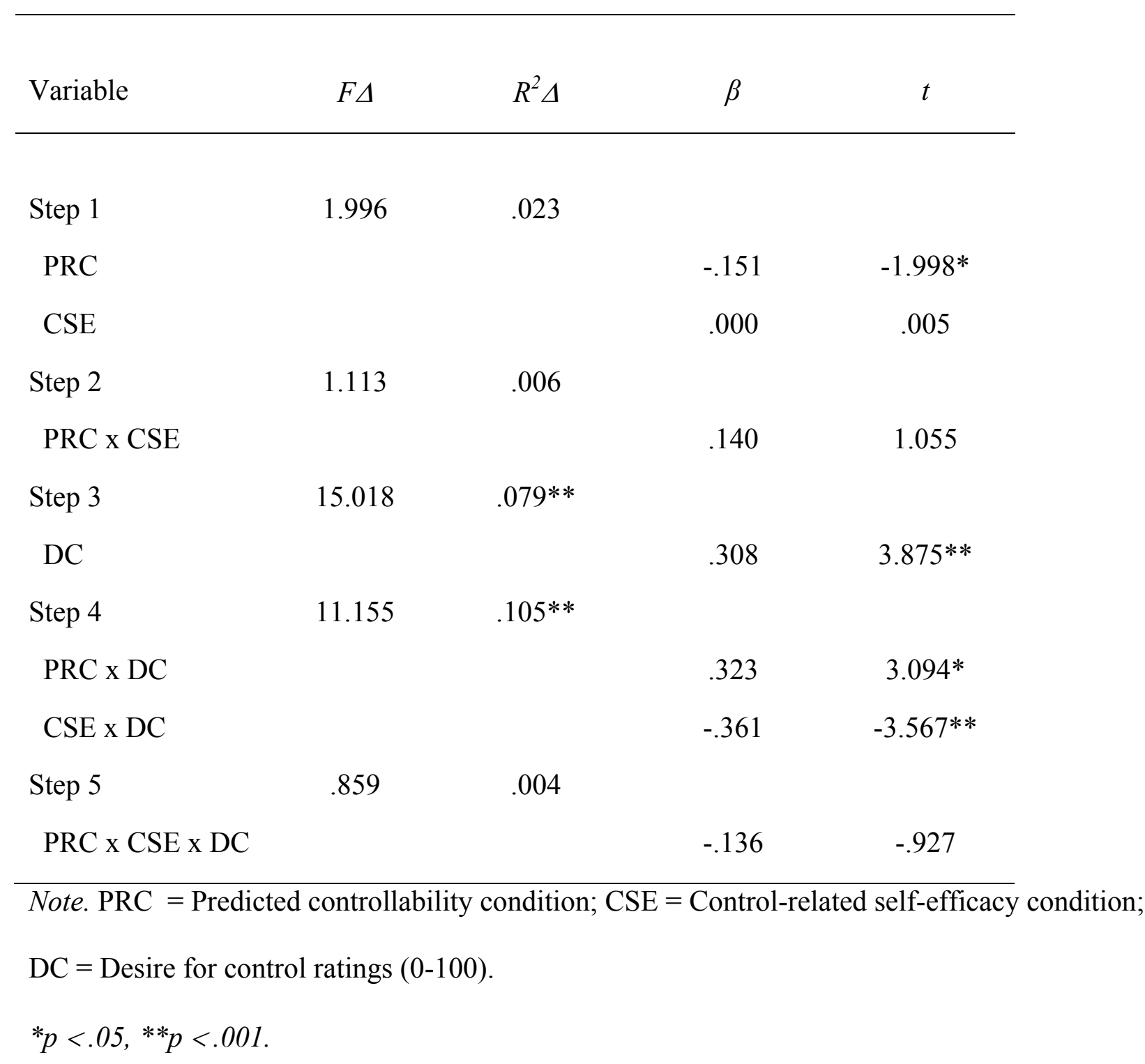




\section{Table 3 .}

Summary of Intercorrelations, Means, and Standard Deviations of Time 2 cleaning (in seconds) for High and Low Conditions of PRC and CSE as a function of DC rating

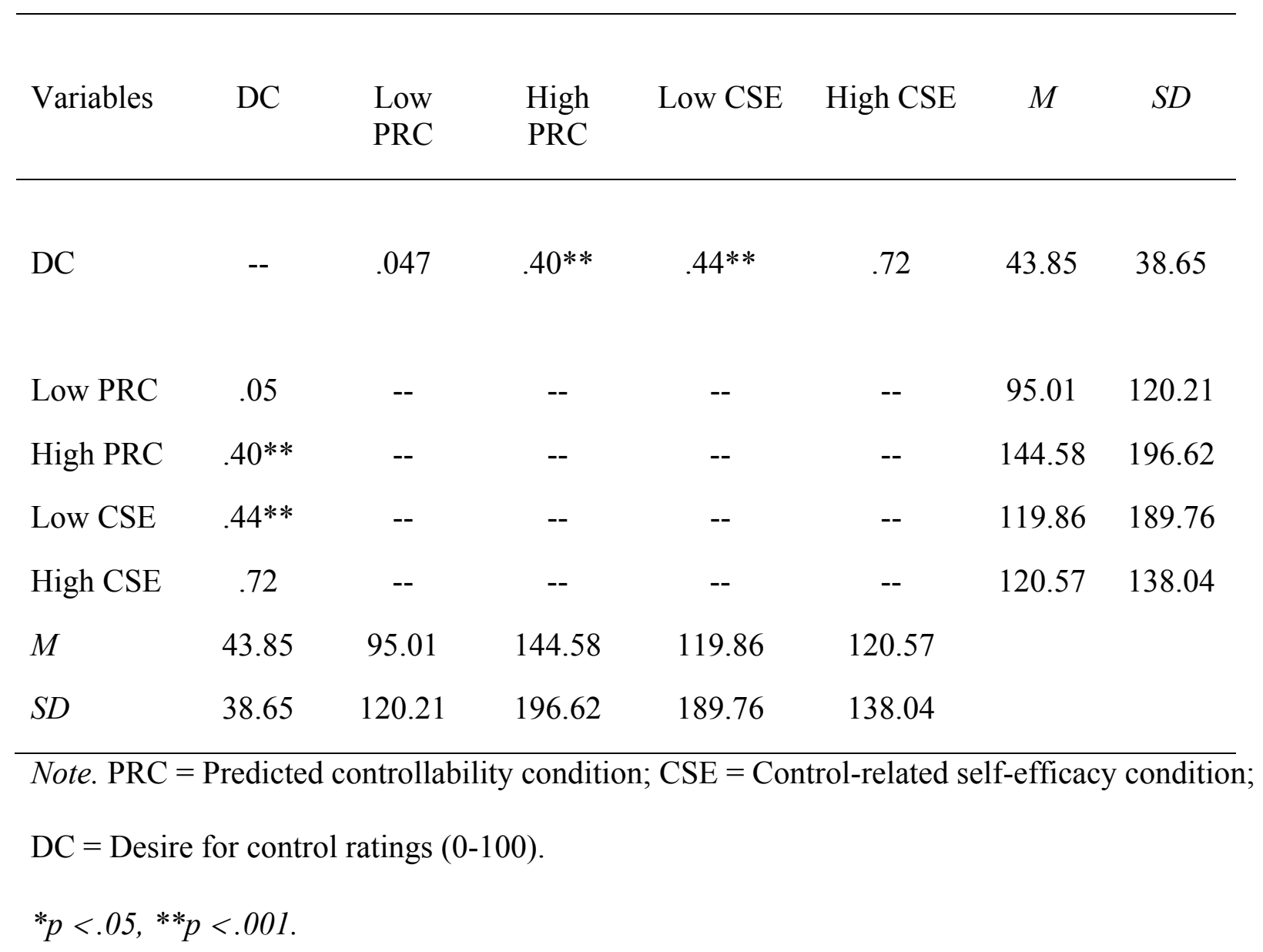




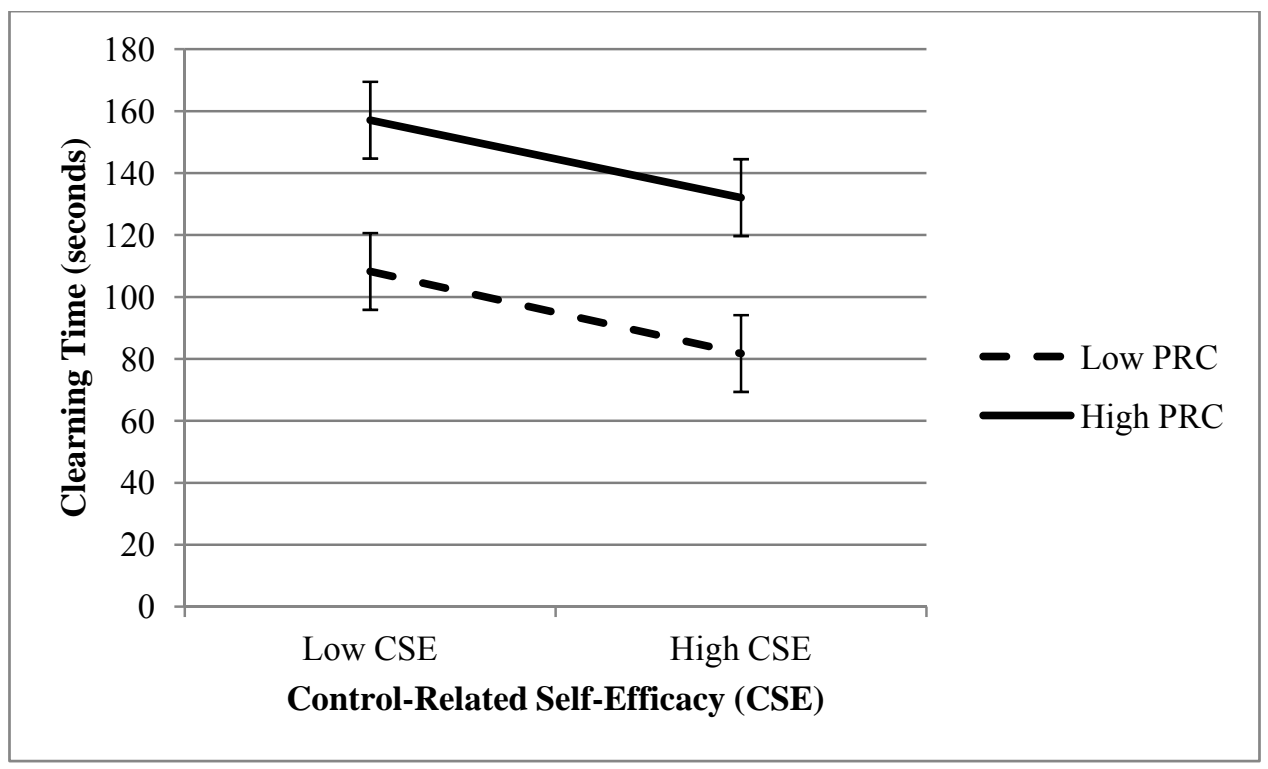

Figure 1. Cleaning time (seconds) at final cleaning period by PRC and CSE. 


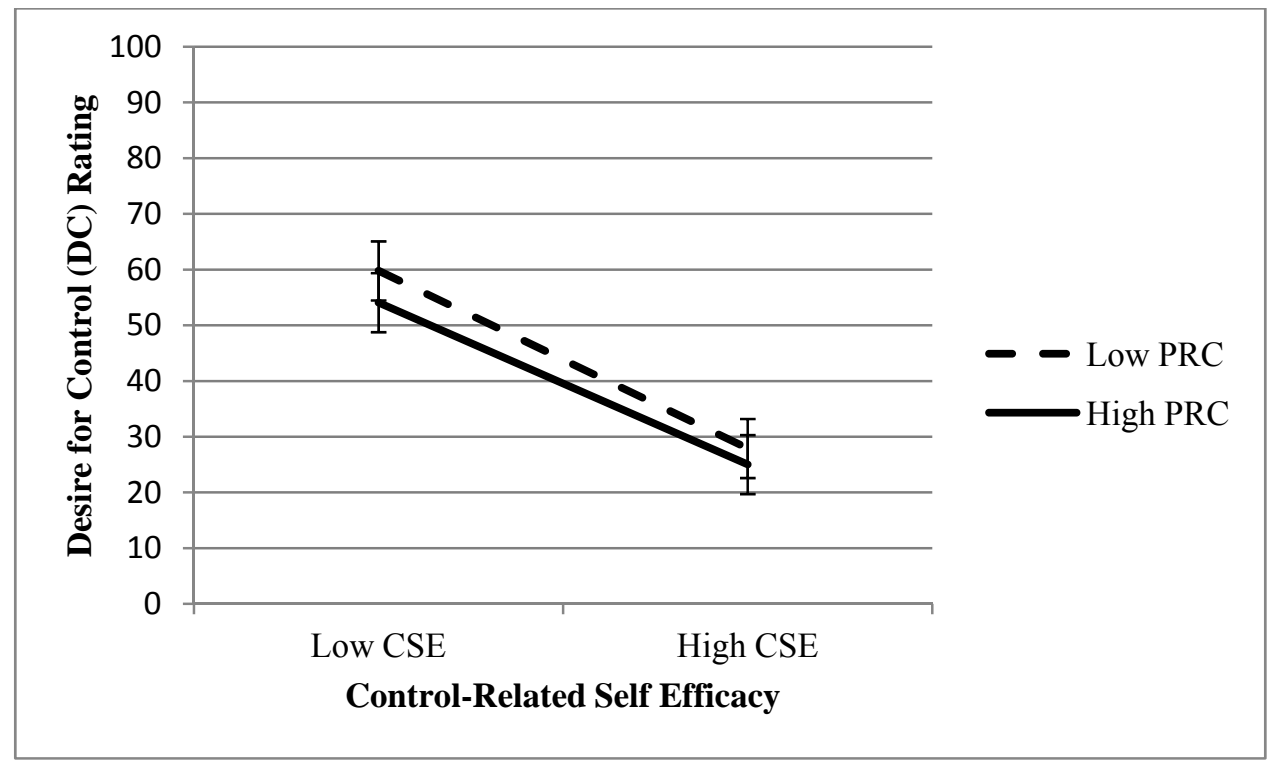

Figure 2. Participants' DC ratings (0-100) by CSE condition. 